\title{
Study on Aerodynamic Characteristics of the Height of Guide Bar of the Simple Rear View
}

\author{
Longcheng Xing*, Linlin Shen, Xiaodong Pan, Jingbo Zhou, Fuqiang Sun \\ College of Civil Engineering and Architecture, Hebei University, Baoding, China \\ Email: xinglongcheng0902@163.com
}

\begin{abstract}
In this paper, the flow field and aerodynamic noise of a simple automotive rearview mirror are analyzed by using Star CCM + software. The external flow field and aerodynamic noise of the rearview mirror are studied by adding a guide bar to the mirror model. The variation of the external flow field and the noise of the rearview mirror are studied by changing the height of the guide $\operatorname{bar}(3 \mathrm{~mm}, 6 \mathrm{~mm}, 10 \mathrm{~mm})$. The results show that the pressure difference between the windward side and the leeward side of the rearview mirror is an important factor causing the airflow resistance. The location of the surface sound source of the rearview mirror is mainly concentrated in the A-pillar area. The rear-view mirrors with $10 \mathrm{~mm}$ guide bar weaken the pressure gradient generated by the airflow through the rearview mirror, and the airflow separation and re-attachment are small, which significantly deviates from the support facing the vortex position of the vehicle outflow field, reducing the effect of turbulent pulsating pressure on the support surface, and the pressure pulsation is superior to the other two cases.
\end{abstract}

Keywords:Rearview mirror, aerodynamic characteristics, aerodynamic noise.

\section{Introduction}

With the improvement of people's living standards, automobiles have become more and more important in our daily life, and people's requirements for the comfort of automobiles in the process of driving are also getting higher and higher. Noise characteristics of modern automobiles are one of the important symbols to measure the quality of a car and the noise inside a car is also one of the main factors affecting the comfort performance of a car. Especially for cars, the noise inside a car is one of the important standards to measure a car grade. Automobile noise is what we do not like when we are driving. Automobile noise has a profound impact on human health, which has attracted the attention of governments all over the world. For high-speed vehicles, aerodynamic noise becomes the main source of noise [1]. A considerable part of the aerodynamic noise can be isolated and absorbed by various interior materials with excellent performance. However, due to the position and special structure of the rear-view mirror outside the vehicle and the A-pillar, they can cause complex turbulent flow in the external flow field of the vehicle, and produce more intense pressure fluctuations, resulting in aerodynamic noise of the vehicle. The contribution to the aerodynamic noise of vehicles is large. The results show that, in the process of vehicle driving, the high-speed air flow separates in the rearview mirror-A pillar region and creates a long tail vortex in the rear of the rearview mirror because the rearview mirror is a blunt body with large structure size exposed to the high-speed airflow [2], which causes a severe pressure pulsation on the side window surface. When the driving speed of the car increases $10 \mathrm{~km} / \mathrm{h}$, the sound pressure level will increase by about $2.5 \mathrm{~dB}$, that is, the aerodynamic noise generated by the rearview mirror is approximately proportional to the sixth power of the speed [3]. Therefore, the research on the flow field characteristics and the generating principle of aerodynamic noise near the rearview mirror can provide a strong support for the noise reduction. The guide bar is a long strip of bump added to the windward side of the rearview mirror cover. When the car is running at high speed, when the air flows through the rearview mirror, it can separate the air flow, so that the re-attachment of the air in the car side window is reduced. Thus, the pressure gradient change on the rear side of the rearview mirror is reduced, and the turbulence region is deviated from the side window outward, which reduces the turbulence fluctuation pressure change caused by the rearview mirror and improves the sound source distribution. 
Chen Xin and Gao Changfeng [4] used two methods of experiment and simulation, and applied the principle of bionic noise reduction to analyze the influence of bionic rear-view mirror on aerodynamic noise. It is concluded that the rear edge modeling of the bionic owl wing can effectively reduce the aerodynamic noise of the rear view mirror. Based on the knowledge of aeroacoustics and aerodynamics, Fu Limin and Zhang Lili [5] analyzed the characteristics of the external flow field, the theory of aerodynamic noise and its generation principle, and the relationship between noise and fluctuating pressure. It is concluded that there is intense eddy flow near the side window surface and A-pillar, and the fluctuating pressure is very high. Therefore, the aerodynamic noise outside the automobile is mainly affected by the rearview mirror. Yang Bo and Huang Lina[6] simulated the aerodynamic noise near the A-pillar of the rearview mirror, and got the effect of mesh fineness on the simulation accuracy of aerodynamic noise. The relationship between fluctuating pressure and aerodynamic noise was studied by collecting the static pressure of each monitoring point. Li Qiliang[7] of Tongji University analyzed and studied the influence of different parameters of rearview mirrors on aerodynamic noise by numerical calculation method based on the general rearview mirror shape. Mingde su and Jiun-der Yu proposed a parallel large eddy simulation of unstructured meshes. They propose a new model that can use structured or unstructured meshes. They develop models and algorithms that can be used to simulate turbulence in a car's rearview mirror. In 1986, DobrxynskiW studied the contribution of aerodynamic noise sources in different parts of the vehicle to the total noise of the vehicle, and concluded that the contribution of the ventilation window and the front side window to the overall noise of the vehicle was the largest.

In 1990, George[8], an American scholar, systematically expounded the origin and principle of automobile aerodynamic noise, pointing out that aerodynamic noise is caused by the fluctuating pressure of the air outside the vehicle, and that the aerodynamic noise inside and outside the vehicle is greatly affected by the distribution of the fluctuating pressure outside the vehicle. Beginning in the early 80 , with the development of computer level and computational fluid dynamics (CFD), the CFD method began to develop gradually in the field of automotive engineering, especially in the field of automotive aerodynamics. Due to the convenience and rapidity of computer simulation, it is not limited by the wind tunnel, as well as the limitations of various practical parameters. Therefore, CFD has become an important research tool.

Therefore, the CFD method has been combined with the experimental research method (experimental Fluid dynamics) and the theoretical research method (theoretical Fluid dynamics) to become the three research methods in the field of fluid mechanics. Despite the tireless efforts of scientific researchers, the research on aerodynamic noise of automobiles has been greatly developed[9-11]. Because the research time is short and the problem of itself is more complicated, the research work in aerodynamic noise has much to do. We also need our unremitting efforts to measure and estimate the aerodynamic noise and the analysis and test method of the aerodynamic noise in the vehicle outflow field.

\section{Theory of Aerodynamic Noise and Flow Field}

\subsection{Generation and Distribution of Aerodynamic Noise Around Vehicle}

Aerodynamic noise is a kind of noise with random frequency and amplitude, which is produced directly by airflow. Fundamentally speaking, the aerodynamic noise inside and outside the vehicle is caused by pressure pulsation and unstable flow of air near the vehicle. The basic cause of Automotive Aerodynamic noise caused by local air separation is the strong fluctuating turbulent pressure around the body and protruding parts. There are two types of turbulent pressure, one is to ensure the safety of driving, comfort and other accessories such as rearview mirror; the other is related to the geometric parameters of the body characteristics.

The aerodynamic noise of automobiles is mainly in the following ways.

Wind Noise: When the airflow passes through a slender object, the wake will produce Karman Vortex. The resulting wind noise mainly affects the low frequency domain noise, seriously affecting the level of noise in the car.

Wind turbulence noise: after passing through the body surface, the airflow is turbulent. Turbulence causes constant and periodic changes in the pressure on the surface of the vehicle over time. Under this excitation, the panels on the windows and body surface vibrate, causing noise and transmitting noise to the vehicle. This kind of aerodynamic noise is called wind-induced noise. The main research content of this 
paper is the wind-induced noise, because the wind-induced noise generated mainly in the A-pillar and the rearview mirror area will seriously affect the vehicle noise level in high-speed motion. The wind turbulence noise belongs to the broadband noise in frequency band noise, and the frequency is from $20-6000 \mathrm{~Hz}$.

Edge noise: Because of the existence of some sharp edges and corners on the body surface, which leads to two-dimensional fluid separation flow, resulting in aerodynamic noise for the edge noise.

\subsection{Correlation Theory of Aerodynamic Acoustic Wave Equation}

\subsubsection{Basic equations of fluid motion}

Any fluid is in accordance with the law of conservation, the basic Conservation law is: the Law of mass conservation, momentum, conservation of energy. Here we will briefly introduce the basic equations of fluid motion.

Momentum equation:

$$
\rho \frac{\mathrm{d} u}{d t}=\rho f-\nabla p+\nabla \tau
$$

energy equation:

$$
\rho \frac{d e}{d t}=-\nabla q+W_{v}+E_{k}+Q_{v}+\Phi+\frac{\partial p}{\partial t}
$$

continuity equation:

$$
\frac{\partial \rho}{\partial \mathrm{t}}+\nabla \rho u=0
$$

state equation:

$$
f\left(\mathrm{p}, \rho, T_{0}\right)=0
$$

Navier Stokes equation:

$$
\rho \frac{\mathrm{d} u_{i}}{d t}=\rho f_{i}-\frac{\partial}{\partial x_{i}}\left(p+\frac{2}{3} \mu \nabla u\right)+\frac{\partial}{\partial x_{j}}\left[\mu\left(\frac{\partial u_{i}}{\partial x_{j}}+\frac{\partial u_{j}}{\partial x_{i}}\right)\right]
$$

The meanings of symbols in the form are as follows:

$\rho$ - Fluid density. T——Time.

$\nabla$ - - Hamilton operator, $\nabla=\vec{i} \frac{\partial}{\partial x_{1}}+\vec{j} \frac{\partial}{\partial x_{2}}+\vec{k} \frac{\partial}{\partial x_{3}}$

$x_{1}, x_{2}, x_{3}$ - The three coordinate variables of the Descartes coordinate system, abbreviated to $x_{i}$ and $x_{j}$.

$i, j$ - Subscript, $i, j=1,2,3$

$i, j, k$ - They are the unit vectors in the direction $x_{1}, x_{2}, x_{3}$

$u$ - Fluid velocity vector, its component in the direction $x_{i}$ is $u_{j}$.

$f$ - Volume forces acting on fluids. $p-$ Pressure in flow field.

\subsubsection{Wave equation of sound in motion fluid}

The acoustic radiation equation of the moving fluid can be derived from the Navier Stokes equation. In 1952, Lighthill deduced the wave equation of fluid sounding based on N S equation and continuous equation.

For convenience, let's first express the continuity equation and the momentum equation in tensor form.

From the formula $(2-3) \frac{\partial \rho}{\partial \mathrm{t}}+\nabla \rho u=0$

It can be obtained

$$
\frac{\partial \rho}{\partial t}+\frac{\partial \rho_{i}}{\partial x_{i}}=0
$$

After the volumetric force is omitted, it can be obtained by N-S equation 


$$
\begin{gathered}
\frac{\partial \rho u_{i}}{\partial t}+\frac{\partial \rho u_{i} u_{j}}{\partial x_{j}}=-\frac{\partial p}{\partial x_{i}}+u \frac{\partial}{\partial x_{j}}\left(\frac{\partial u_{i}}{\partial x_{j}}+\frac{\partial u_{j}}{\partial x_{i}}-\frac{2}{3} \delta_{i j} \frac{\partial u_{k}}{\partial x_{k}}\right) \\
e_{i j}=u \frac{\partial}{\partial x_{j}}\left(\frac{\partial u_{i}}{\partial x_{j}}+\frac{\partial u_{j}}{\partial x_{j}}-\frac{2}{3} \delta_{i j} \frac{\partial u_{k}}{\partial x_{k}}\right)
\end{gathered}
$$

which makes $e_{i j}$ the viscous stress tensor.

The formula (2.6) can be abbreviated as

$$
\frac{\partial \rho u_{i}}{\partial t}+\frac{\partial \rho u_{i} u_{j}}{\partial x_{j}}=-\frac{\partial p}{\partial x_{i}}+\frac{\partial e_{i j}}{\partial x_{j}}
$$

The formula (2.5) is differential to t, we can get

The (2.8) divergence can be obtained.

$$
\frac{\partial^{2} \rho}{\partial t^{2}}+\frac{\partial^{2}\left(\rho u_{i}\right)}{\partial x_{i} \partial t}=0
$$

$$
\frac{\partial^{2} \rho u_{i}}{\partial x_{i} \partial t}+\frac{\partial^{2} \rho u_{i} u_{j}}{\partial x_{i} \partial x_{j}}=-\frac{\partial^{2} p}{\partial x_{i}^{2}}+\frac{\partial^{2} e_{i j}}{\partial x_{i} \partial x_{j}}
$$

The formula (2.9) is subtracted from (2.10).

$$
\frac{\partial^{2} \rho}{\partial t^{2}}=\frac{\partial^{2} p}{\partial x_{i}^{2}}+\frac{\partial e_{i j}}{\partial x_{i} \partial x_{j}}\left(\rho u_{i} u_{j}-e_{i j}\right)
$$

Both sides of the upper part are subtracted from $c_{0}^{2} \nabla^{2} \rho$,

$$
\frac{\partial^{2} \rho}{\partial t^{2}}-c_{0}^{2} \nabla^{2} \rho=\frac{\partial^{2} p}{\partial x_{i}^{2}}+\frac{\partial e_{i j}}{\partial x_{i} \partial x_{j}}\left(\rho u_{i} u_{j}-e_{i j}\right)-c_{0}^{2} \nabla^{2} \rho=\frac{\partial^{2}}{\partial x_{i} x_{j}}\left[\rho u_{i} u_{j}-e_{i j}+\delta_{i j}\left(p-c_{0}^{2} \rho\right)\right]
$$

Supposing

$$
T_{i j}^{\prime}=\frac{\partial^{2}}{\partial x_{i} \partial x_{j}}\left[\rho u_{i} u_{j}-e_{i j}+\delta_{i j}\left(p-c_{0}^{2} \rho\right)\right]
$$

The formula (2.12) can be abbreviated as

$$
\frac{\partial^{2} \rho}{\partial t^{2}}-c_{0}^{2} \nabla^{2} \rho=\frac{\partial^{2} T_{i j}^{\prime}}{\partial x_{i} \partial x_{j}}
$$

We can see that both $\frac{\partial \rho_{0}}{\partial t}$ and $\frac{\partial^{2} \rho_{0}}{\partial x_{i} \partial x_{j}}$ should be zero, so we can get density wave equation.

$$
\frac{\partial^{2} \rho^{\prime}}{\partial \tau^{2}}-c_{0}^{2} \nabla^{2} \rho^{\prime}=\frac{\partial^{2} T_{i j}}{\partial y_{i} \partial y_{j}}
$$

where:

$T_{i j}=\rho u_{i} u_{j}-e_{i j}+\delta_{i j}\left(p^{\prime}\right)-c_{0}^{2} \delta_{i j}\left(p^{\prime}\right)$ is Lighthill tensor;

$u$ is fluid velocity; $e_{i j}$ is viscous stress tensor; $\delta_{i j}$ is unit tensor;

$\rho$ is fluid density; $c_{0}$ is Velocity.

$\rho_{0}$ is the mean value of the undisturbed fluid density or fluid density;

$\rho^{\prime}$ is fluctuation of fluid density, $\rho^{\prime}=\rho-\rho_{0}$;

$p$ is pressure in the Flow field;

$p_{0}$ is the flow field pressure when undisturbed or the mean value of the pressure in the flow field;

$p^{\prime}$ is the fluctuation of pressure in the flow field, $p^{\prime}=p-p_{0}$.

In order to obtain a vehicle-induced aerodynamic noise wave equation which is suitable for the driving process, a coordinate $y^{\prime}$ with the medium motion is introduced first. That is 


$$
y^{\prime}=y_{i}-\delta_{1 i} U \tau
$$

Since the Lighthill equation is derived from the continuity equation and the momentum equation, and the momentum equation is invariant under the Galileo transformation, the form of the Lighthill equation under the motion coordinates is still

and

$$
\frac{\partial^{2} \rho^{\prime}}{\partial \tau^{2}}-c_{0}^{2} \nabla^{2} \rho^{\prime}=\frac{\partial^{2} T_{i j}^{\prime}}{\partial y_{i}^{\prime} \partial y_{j}^{\prime}}
$$

The equation in the form of a fixed coordinate is:

$$
\begin{aligned}
& T_{i j}^{\prime}=\rho u_{i}^{\prime} u_{j}^{\prime}-e_{i j}+\delta_{i j}\left(p-p_{0}\right)-c_{0}^{2} \delta_{i j}\left(\rho-\rho_{0}\right) \\
& u_{i}^{\prime}=u_{i}-\delta_{1 i} U
\end{aligned}
$$

$$
\frac{D_{0}^{2} \rho^{\prime}}{D \tau^{2}}-c_{0}^{2} \nabla^{2} \rho^{\prime}=\frac{\partial^{2} T_{i j}^{\prime}}{\partial y_{i} \partial y_{j}}
$$

This is a general aerodynamic noise wave equation, also known as the generalized Lighthill equation, which is suitable for vehicles in the process of running.

\section{$3 \quad$ Modeling and Simulation}

\subsection{Basic Parameters}

In this paper, the aerodynamic noise of the rearview mirror is studied by changing the height of the guide bars $(3 \mathrm{~mm}, 6 \mathrm{~mm}, 10 \mathrm{~mm})$ on a simple rearview mirror model. The guide bar is chamfered. The guide bar is added to the windward surface of the simple rearview mirror model. When the airflow passes through the rearview mirror, it can act as a shunt, thus reducing the turbulent motion behind the rearview mirror and the pressure pulsation, thus reducing the aerodynamic noise in the rearview mirror area. Finally, a reasonable model of rearview mirror is obtained, which provides technical support for the development of aerodynamic noise of rearview mirror. A semi-cylinder and a quarter sphere are used to simulate the rearview mirror. As shown in the figure 3-1.The diameter of the sphere is $D=0.2 \mathrm{~m}$ and the total height of the rearview mirror is $\mathrm{H}=0.3 \mathrm{~m}$. The rearview mirror is directly installed in the cuboid computing domain, with a length of $5 \mathrm{~m}$, a width of $2.4 \mathrm{~m}$, and a high of $1.6 \mathrm{~m}$. The rearview mirror is $0.9 \mathrm{~m}$ from the entrance. Thinking of the support surface as the side window of the car as shown in the figure 3-2 below.

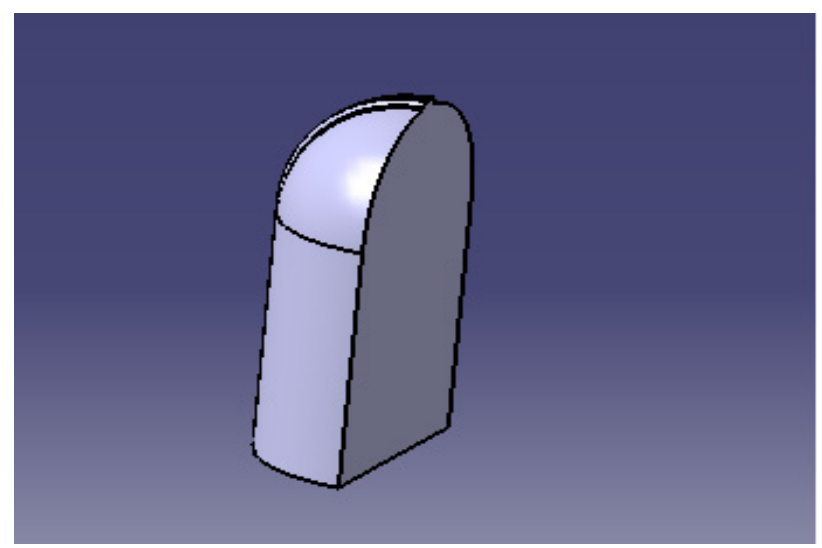

Figure 3-1. Simple rearview mirror 


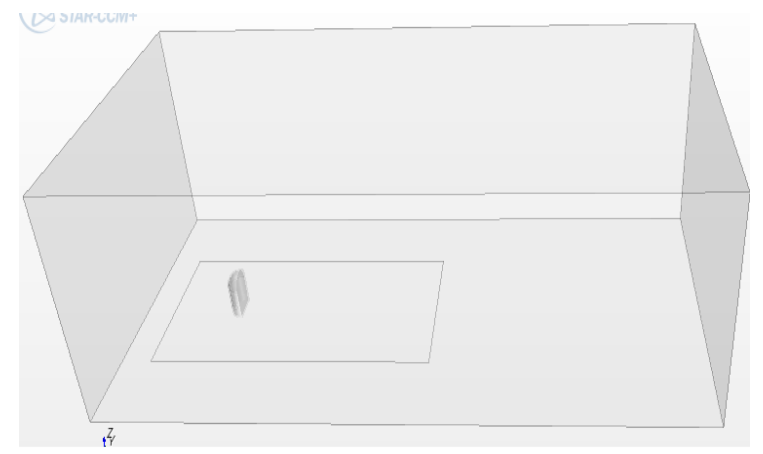

Figure 3-2. Schematic diagram of computational domain

To facilitate our analysis of the data obtained, we establish two sections as shown

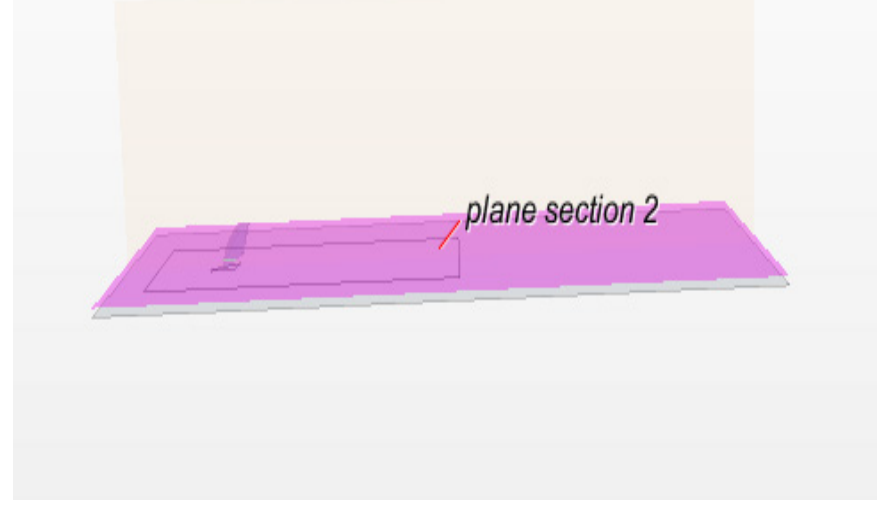

Figure 3-3. the first section

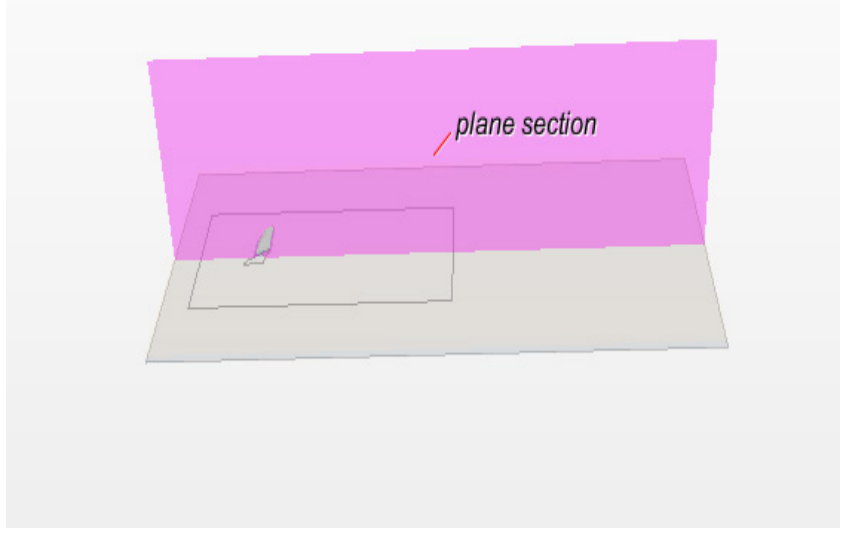

Figure 3-4. the second section

\section{Results Analysis}

\subsection{Velocity Vector Graph}

The velocity vector graphs on the two sections of the guide bar height of $3 \mathrm{~mm}$ are shown below. 


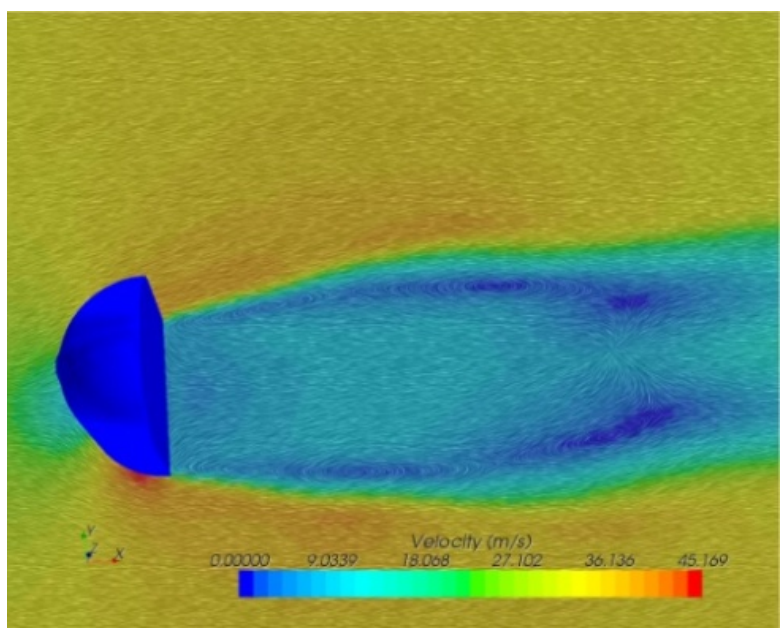

Figure 4-1. The first section velocity vector diagram

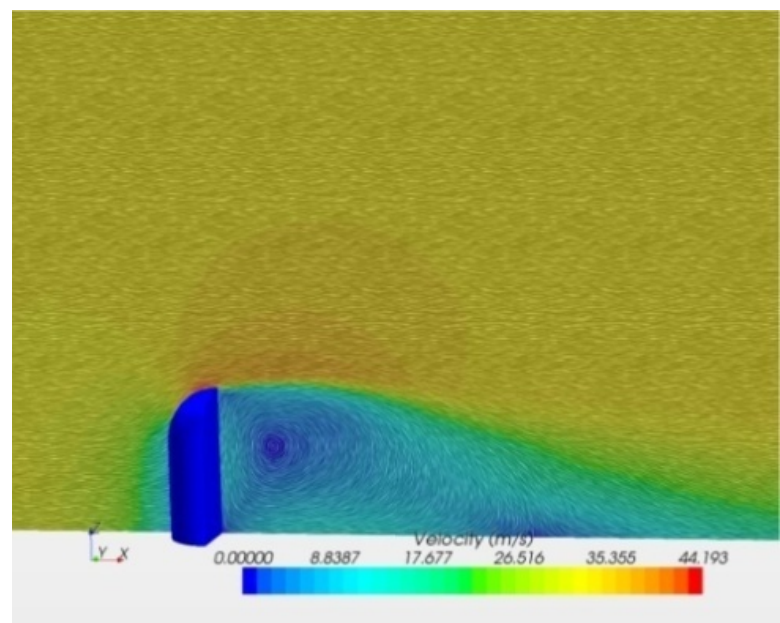

Figure 4-2. the second section velocity vector diagram

The velocity vector graphs on the two sections of the guide bar height of $6 \mathrm{~mm}$ are shown below.

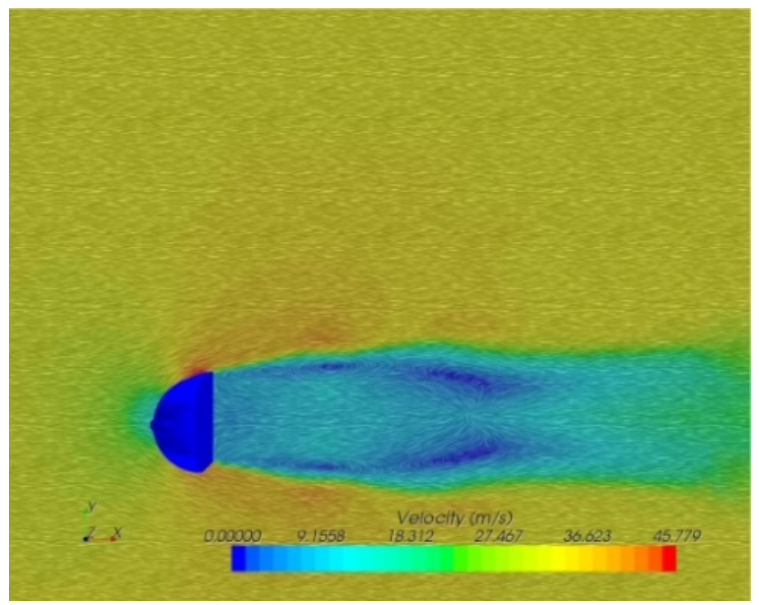

Figure 4-3. the second section velocity vector diagram 


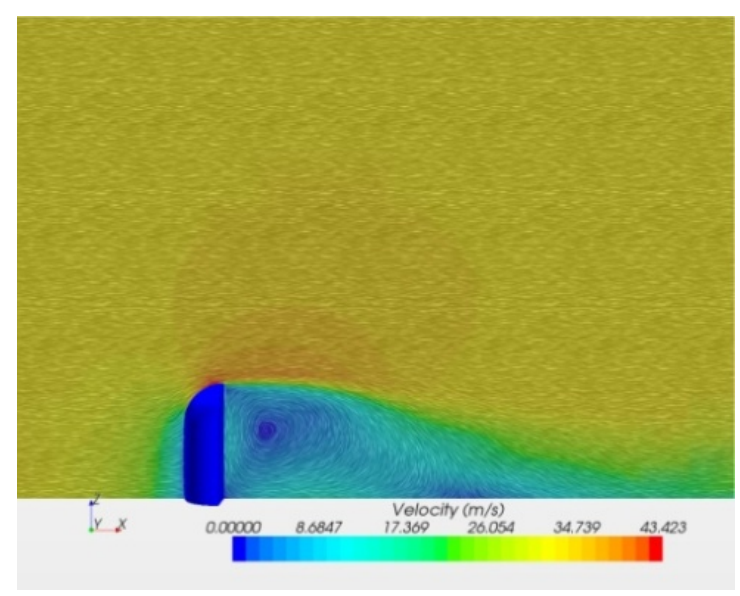

Figure 4-4. the second section velocity vector diagram

The velocity vector graphs on the two sections of the guide bar height of $10 \mathrm{~mm}$ are shown below.

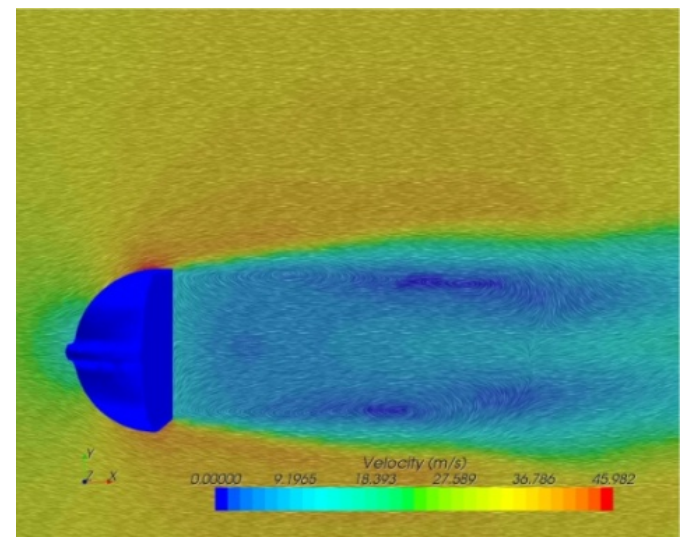

Figure 4-5. The first section velocity vector diagram

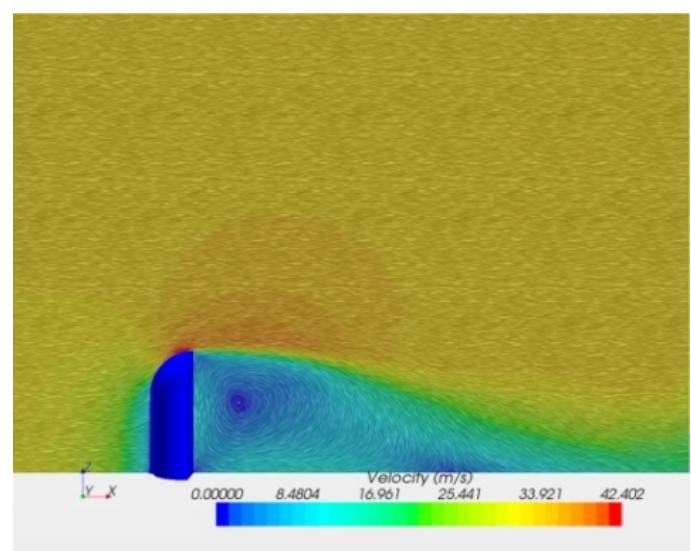

Figure 4-6. the second section velocity vector diagram

From the above pictures, we can see that the maximum position of the airflow velocity of each rearview mirror appears near the windward side. And there are two or four vortices behind the rearview mirror. This is because the hindrance of the rearview mirror affects the movement of the air flow, and the turbulence is formed behind the rearview mirror. The maximum velocity in the first section is greater than the maximum velocity in the second section. The guide bar weakens the aerodynamic noise caused by the turbulent pressure pulsation generated by the rearview mirror directly acting on the side window glass. 
When the height of the guide bar is different, the higher the height of the guide bar is, the place where the vortex occurs is relatively farther away from the rearview mirror.

\subsection{Streamline Diagram of the Second Section}

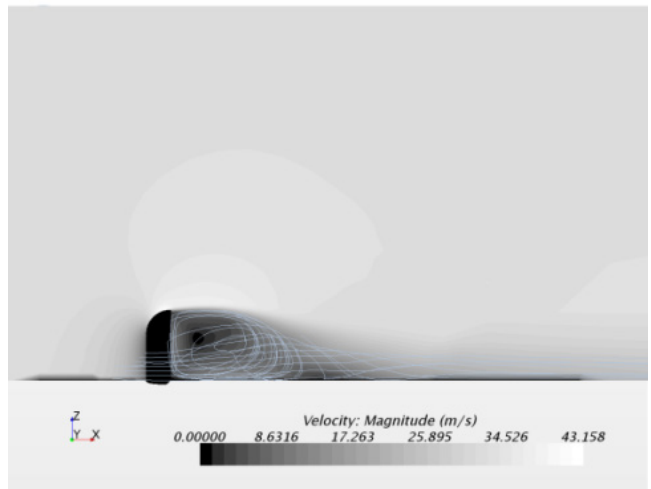

Figure 4-7. The second section average velocity streamline diagram at $3 \mathrm{~mm}$ of guide bar height

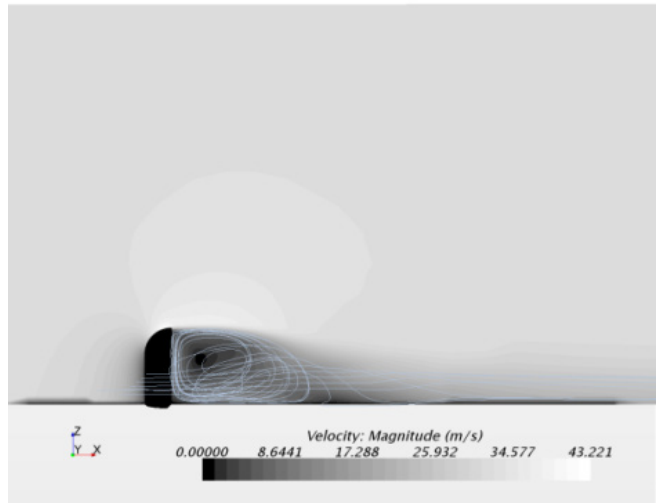

Figure 4-8. The second section average velocity streamline diagram at $6 \mathrm{~mm}$ of guide bar height

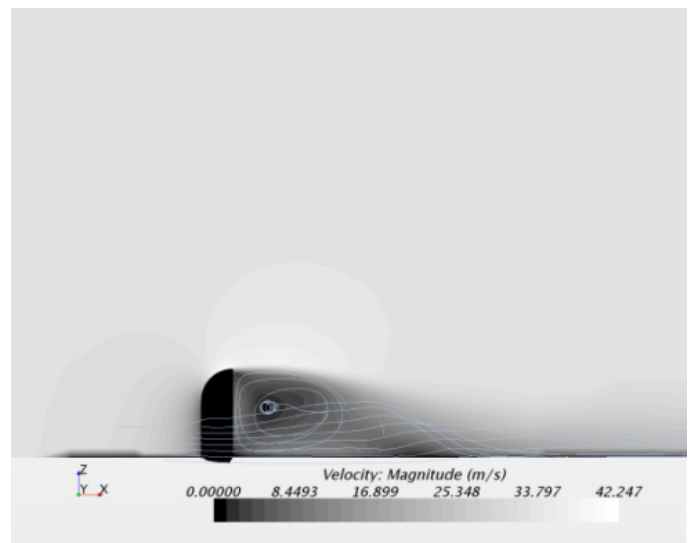

Figure 4-9. The second section average velocity streamline diagram at $10 \mathrm{~mm}$ of guide bar height

As can be seen from the above pictures, the air flow head-on, the flow of the fluid under the rearview mirror is chaotic, the velocity direction changes dramatically, and it is easy to produce strong pressure pulsation. Due to the obstruction of the rearview mirror, a large area of low-speed region appears in its tail, and the air velocity around it is larger, the high-speed air continuously supplements the low-speed air flow to form a counter-current, resulting in the appearance of vortex. This is similar to the situation in which 
the leaves fly with the air as the car flies through the bottom of the fallen leaves at high speed in life. By comparing the above figure, when the higher the height of the guide bar, the smaller the vortex behind the rear-view mirror, the farther away from the support surface.

\subsection{Pressure Cloud}

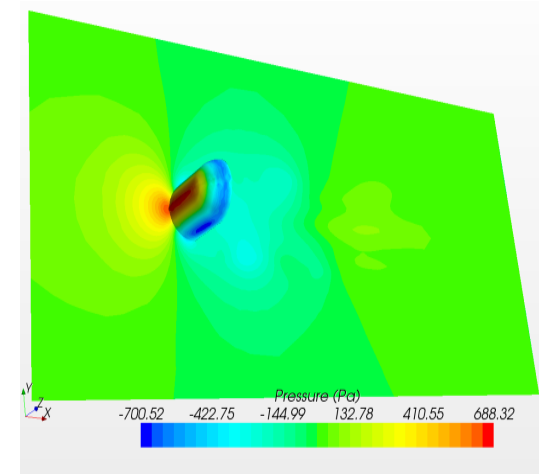

Figure 4-10. Pressure cloud image at 3mm of guide bar height

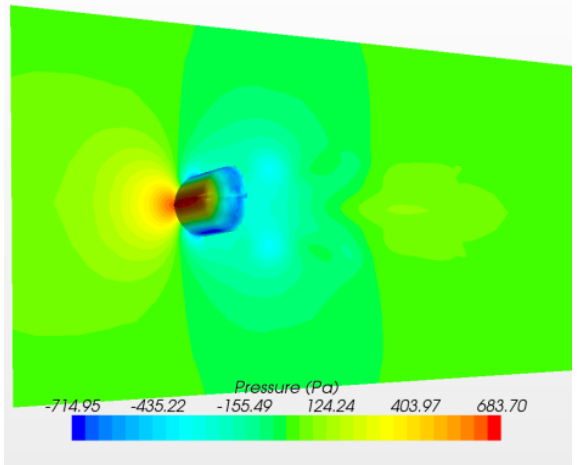

Figure 4-11. Pressure cloud image at $6 \mathrm{~mm}$ of guide bar height

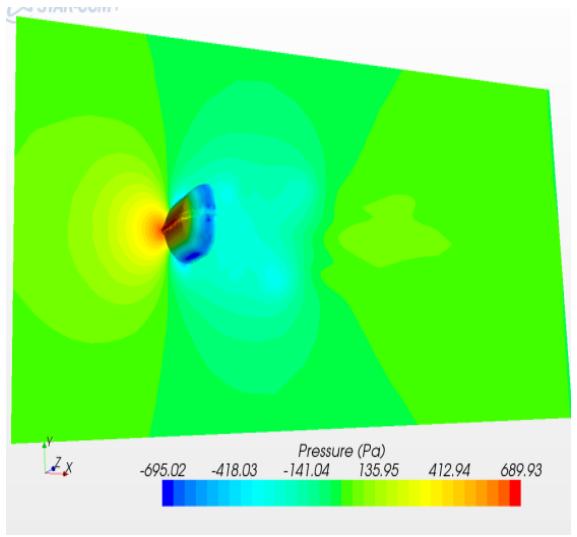

Figure 4-12. Pressure cloud image at $10 \mathrm{~mm}$ of guide bar height

As can be seen from the above diagram, the positive pressure value of the rear view mirror is larger on the windward side, and the negative pressure value on the leeward side is larger. The pressure difference between the windward side and the leeward side is the important factor that causes the vehicle running resistance. From the guide bar height, the drag coefficient of the $10 \mathrm{~mm}$ rearview mirror is the smallest, which reduces the fuel consumption in the process of driving. The pressure gradient of the rearview mirror with $10 \mathrm{~mm}$ tendons is less changed, and the air separation and reattachment degree are smaller. 


\subsection{Turbulent Kinetic Energy Diagram}

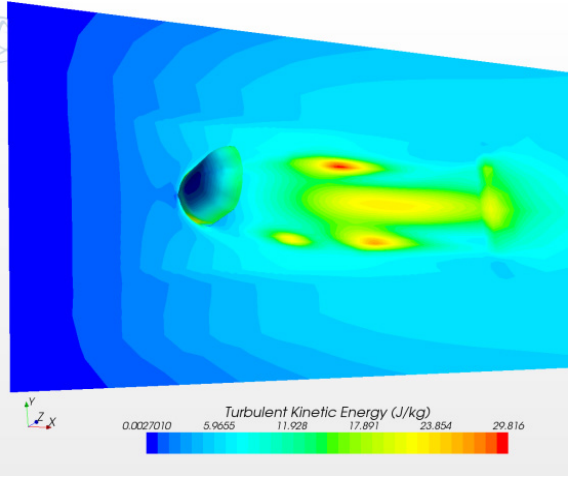

Figure 4-13. Turbulence kinetic energy distribution nephogram with a guide bar height of $3 \mathrm{~mm}$

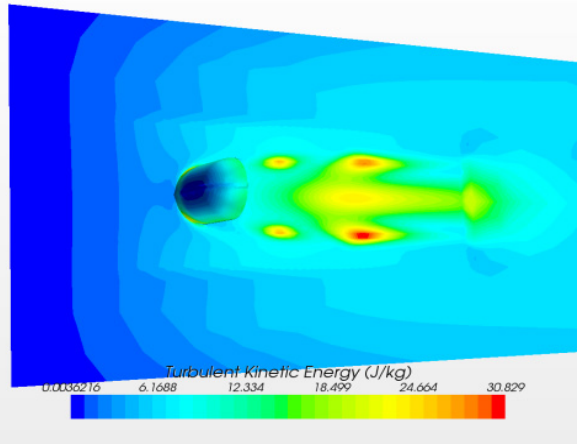

Figure 4-14. Turbulence kinetic energy distribution nephogram with a guide bar height of $6 \mathrm{~mm}$

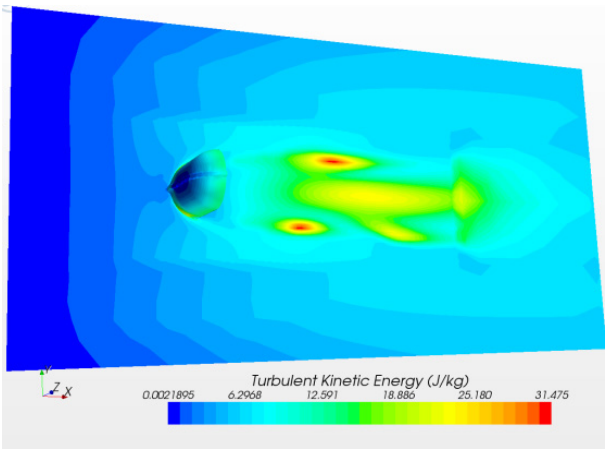

Figure 4-15. Turbulence kinetic energy distribution nephogram with a guide bar height of 10mm

Turbulent energy is considered as a measure of turbulent intensity. The greater the turbulent energy in a region, the more intense the turbulent motion in the region, and the more intense the pressure fluctuation. The airflow passes through the rearview mirror to form turbulence behind it, resulting in higher turbulent kinetic energy. The direction of turbulence is shown in the figure. The rearview mirror with turbulence far away from the rear view mirror has better effect and can reduce noise effectively. From the guide bar height, the turbulent flow energy behind the $10 \mathrm{~mm}$ rearview mirror is the weakest and far from the support surface. The turbulent region deviates from the support surface and the pressure pulsation is lower than the other two cases.

\section{Conclusion}

In this paper, the star ccm + software is used to simulate the flow field and sound field of the rearview 
mirror model. The influence of the stiffened height on the aerodynamic noise of the rearview mirror is studied by stiffening the simple rearview mirror model.

1. The maximum positive pressure of the rearview mirror is located on the windward side, and the negative pressure is generated on the leeward side. The pressure difference between the two sides is an important factor to produce the airflow resistance. The shape change of the rearview mirror has a great influence on the external flow field and aerodynamic noise of the rearview mirror. Because of the movement of airflow, there will be turbulent motion behind the rearview mirror.

2. Rearview mirrors with different heights will affect the vortex flow around the rearview mirror. The aerodynamic noise in the rearview mirror area can be controlled by controlling the height of the guide bar. The rear-view mirrors with $10 \mathrm{~mm}$ guide bar weaken the pressure gradient generated by the airflow through the rearview mirror, and the airflow separation and re-attachment are small, which significantly deviates from the support facing the vortex position of the vehicle outflow field, reducing the effect of turbulent pulsating pressure on the support surface, and the pressure pulsation is superior to the other two cases.

\section{References}

1. Wu Bingping. Development and Present Situation of Vehicle Noise Regulations and NVH Research Methods[J].Technology and Application, 2009(8): 28-29

2. Wang Yiping. Guzhengqi.Yang Xue .Aerodynamic noise simulation analysis and control of minibus rearview mirror[J]. Aeronautical Dynamics Journal, 2009(7): 1576-1583

3. Ye Li. Takashi Kamioka.et al.Evaluation of Aerodynamic Noise Generated in Production Vehicle Using Experiment and Numerical Simulation [C]. SAE Paper No. 2003-01-1314

4. Gao Changfeng. Study on the Influence of Bionic Rearview Mirror Cover on Aerodynamic Noise on the Exterior Flange of the Vehicle[D]. Jilin: Jilin University, 2014

5. Zhang Lili. Numerical simulation study on aerodynamic noise of high speed car[D]. Jilin: Jilin University, 2006

6. Huang Lina. Study on unsteady field and aerodynamic noise in car rearview mirror[D]. Jilin: Jilin University, 2014

7. Li Qiliang. large eddy simulation of unsteady flow field in vehicle rearview mirror [J]. Journal of aerodynamics, 2010 (8)

8. Katsuya Uchida, Kenji Okumura, Aerodynamic Noise Simulation based on Lattice Boltzmann Method (Surface Pressure Fluctuations around A-pillar ), SAE Paper 1999-01-1127

9. Nurul Murad, Jamal Naser. Computational fluid dynamics study of vehicle A-pillar aero-acoustics. Applied Acoustics 74 (2013) 882-896.

10. Christoph Gabriel. Hybrid Simulation Method to Predict low induced Car Cabin Noise. ATZ worldwide, 2015, $117(9): 54-59$.

11. Hannes M.Frank,Claus-Dieter Munz. Direct aeroacoustic simulation of acoustic feedback phenomena on a side-view mirror. Journal of Sound and Vibration, 2016, 371:132-149. 